\title{
EDITORIAL
}

\section{Elbow and finger extension reanimation in cervical cord injuries}

\author{
Ferry Senjaya, MD, ${ }^{1}$ and Rajiv Midha, MD, MSc, FRCSC² \\ ${ }^{1}$ Neurosurgery Department, Siloam Hospitals Simatupang, Pelita Harapan University, Tangerang, Indonesia; and 2Department of \\ Clinical Neurosciences, Hotchkiss Brain Institute, Cumming School of Medicine, University of Calgary, Alberta, Canada
}

$\mathrm{T}$ HE annual incidence of spinal cord injury (SCI) in the US is approximately 40 cases per million of the population, or roughly 12,000 new cases each year, excluding those who die at the scene of the accident. ${ }^{17}$ This devastating injury occurs most commonly in young, previously healthy, and active individuals, with the most frequent neurological deficit at discharge being incomplete tetraplegia (40.6\%). ${ }^{17}$ One of the most devastating effects of SCI at the cervical level is the loss of arm and/ or hand function, and this imposes a significant impact on a patient's independence. ${ }^{9}$ Recovering arm and hand function, even partially, can have an enormous impact on quality of life, because of their invaluable roles in activities of daily living and mobility. ${ }^{9,20}$

The traditional approach in reconstructive surgery for restoring some arm and hand function has consisted of tendon transfers..$^{10,11}$ Nerve transfer, although rarely used to date, is emerging as a viable method for restoration of function following SCI. ${ }^{10,12,19}$ Nerve transfers offer several advantages over tendon transfers. Tendon transfers require significantly more dissection and extended postoperative limb immobilization while the tendon heals. ${ }^{10}$ Reconstruction of finger flexion and extension must be performed in separate phases, due to conflicting positions for postoperative immobilization. ${ }^{18}$ Nerve transfers, on the other hand, require a shorter period and less restrictive immobilization, and minimal loss of donor muscle function. ${ }^{10}$ Reconstruction of finger flexion and extension can be performed at the same time. The tension/insertion balance of the muscle/tendon unit is preserved with nerve transfers because there is no disruption to the insertion or attachment of the muscle in question, therefore maintaining line of pull and excursion, and avoiding scar-induced restrictions to movement. ${ }^{10}$ Nerve transfers also offer a greater functional gain for a given transfer; ${ }^{9-11}$ that is, the transferred nerve, which originally provided innervation to a single muscle, can reinnervate multiple muscles. Lat- er on, with motor re-education and central plasticity, it is possible to activate multiple functions independently by the same nerve that originally controlled a single function. ${ }^{9,10,16}$

Approximately two-thirds of cervical SCI involves the C-5 or C-6 vertebrae, which typically injures the C6-7 spinal cord. ${ }^{8,15,21}$ This often leaves the patient with generally preserved shoulder function as well as elbow flexion. In most cases (82\%), wrist extension is also largely preserved. ${ }^{21}$ Elbow extension is absent or weak. Hand movements are also absent or extremely impaired. Restoration of elbow extension is an integral part of upper-extremity reconstruction, as recovery of this function will improve elbow stability, enable the performance of pressure-relief maneuvers, permit better manual wheelchair propulsion, allow reaching objects above the shoulder level, and enhance the ability for self-transfers., ${ }^{4,8,13,14}$ Finger extension reanimation is also important because this function is required for object acquisition and release. ${ }^{14}$ The common procedure used to restore elbow extension is tendon or muscle transfer of posterior deltoid or biceps muscle to the triceps. ${ }^{13,14}$ However, most patients who underwent these procedures only regained antigravity muscle strength. ${ }^{8}$ This is also the case for common orthopedic procedures to reanimate finger extension. ${ }^{5}$

In this paper, Bertelli and Ghizoni described their pioneering experience in reconstruction of elbow and finger extension using nerve transfers in patients with midcervical SCI. ${ }^{1}$ The authors have done extensive work in this field and have produced several articles (primarily case reports) related to nerve transfers for SCI. ${ }^{2-8}$ This is their first case series reported. They performed a total of 27 nerve transfers, in 13 upper limbs in 7 patients. Divisions of the axillary nerve were used to reinnervate the triceps muscle, and the nerve to the supinator was transferred to the adjacent posterior interosseous nerve to regain thumb and finger extension. The surgery was effective in all 13 
limbs that were operated on, except a failure of the distal transfer for thumb and finger extension in 1 hand.

The authors eloquently discuss their findings and note several important observations. Of great interest is their observation that even in cases in which the axillary nerve was used in its entirety, none of the patients experienced reduction of shoulder function. The authors suggest that this might be the result of compensation from the normally functioning supraspinatus muscle and improved proximal arm usage after surgery. Another important observation is that in patients with C-6 cervical cord injury with already weak pronation, the loss of supination (such as after distal radio-ulnar fusion) does not cause a noticeable deficit or complaints from patients. The resultant forearm stabilization (after fusion) improves finger and thumb extension and therefore better hand function, which outweighs the loss of forearm rotation.

The results are encouraging and the potential for greater use can occur, but with the following caveats. Only patients with complete SCIs should be considered for nerve transfer procedures because patients with incomplete injuries have a variable propensity for recovery. The latter should be serially followed until a plateau of clinical function occurs. It is of paramount importance to do a thorough clinical examination, electrodiagnostic test, and surface electrical stimulation (when available) to differentiate a central or peripheral paralysis in target muscles prior to surgery, especially in patients who present late to the clinic. In patients with central-type paralysis (muscle innervated by infralesional metamere, with preserved lower motor neurons) of the target muscles, nerve transfer may restore these muscles even years after injury, as long as the mechanical properties of the limb, such as joint mobility, are preserved. Brown reported successful nerve transfer procedures to reconstruct elbow extension, wrist flexion-extension, and finger flexion-extension in a patient with tetraplegia 13 years after injury. ${ }^{9}$ Preoperative investigations in that case revealed mostly intact lower motor neuron innervation in the reconstructed upper limb, which might result from a remarkably thin segment of injured metamere. This may be a rare case, but it shows that functional reinnervation in such a situation is possible, contrary to what the authors state in their discussion. The authors in the current paper, however, do stress that surgery should be undertaken within 12 months of injury due to the surprisingly high occurrence of concomitant peripheral-type palsy (as determined by lack of evoked muscle contractions from direct electrical stimulation of the recipient nerve) in 22 of 27 instances. This is presumably because injury at midcervical vertebrae typically injured the C- 6 but also, to a variable extent, the C-7 spinal cord, resulting in direct damage to the motor neuron pool of triceps and extensor digitorum communis muscles, thus producing a peripheral-type paralysis. We would encourage the use of rigorous preoperative electrodiagnostic assessment, with electromyographic recordings of muscles targeted for re-innervation by nerve transfers, to determine their denervated versus intact status. This would augment the intraoperative findings. One clear take-home message from the current paper is that surgery for reanimation can and should be performed in cases in which either pre- or intraoperative electrical studies demonstrate peripheral-type palsy. Simply, injury to the next caudal metameric segment is not a contraindication to surgery but rather can be considered an indication for surgery once the patient is deemed clinically suitable for an operation at the 6- to 12-month interval from initial SCI.

The authors are to be commended for their relentless work in this field. Hopefully, this article will spark a greater interest in neurosurgeons in peripheral nerve surgery and its role for peripheral neural reconstruction, to improve functionality in SCI in particular.

http://thejns.org/doi/abs/10.3171/2014.5.JNS14964

\section{References}

1. Bertelli JA, Ghizoni MF: Nerve transfers for elbow and finger extension reconstruction in midcervical spinal cord injuries. J Neurosurg [epub ahead of print October 24, 2014. DOI: 10.3171/2014.8.JNS14277]

2. Bertelli JA, Ghizoni MF: Single-stage surgery combining nerve and tendon transfers for bilateral upper limb reconstruction in a tetraplegic patient: case report. J Hand Surg Am 38:1366-1369, 2013

3. Bertelli JA, Ghizoni MF: Transfer of nerve branch to the brachialis to reconstruct elbow extension in incomplete tetraplegia: case report. J Hand Surg Am 37:1990-1993, 2012

4. Bertelli JA, Ghizoni MF, Tacca CP: Transfer of the teres minor motor branch for triceps reinnervation in tetraplegia. Case report. J Neurosurg 114:1457-1460, 2011

5. Bertelli JA, Kechele PR, Santos MA, Besen BAMP, Duarte $\mathrm{H}$ : Anatomical feasibility of transferring supinator motor branches to the posterior interosseous nerve in C7-T1 brachial plexus palsies. Laboratory investigation. J Neurosurg 111:326-331, 2009

6. Bertelli JA, Mendes Lehm VL, Tacca CP, Winkelmann Duarte EC, Ghizoni MF, Duarte H: Transfer of the distal terminal motor branch of the extensor carpi radialis brevis to the nerve of the flexor pollicis longus: an anatomic study and clinical application in a tetraplegic patient. Neurosurgery 70:1011-1016, 2012

7. Bertelli JA, Tacca CP, Ghizoni MF, Kechele PR, Santos MA: Transfer of supinator motor branches to the posterior interosseous nerve to reconstruct thumb and finger extension in tetraplegia: case report. J Hand Surg Am 35:1647-1651, 2010

8. Bertelli JA, Tacca CP, Winkelmann Duarte EC, Ghizoni MF, Duarte H: Transfer of axillary nerve branches to reconstruct elbow extension in tetraplegics: a laboratory investigation of surgical feasibility. Microsurgery 31:376-381, 2011

9. Brown JM: Nerve transfers in tetraplegia I: background and technique. Surg Neurol Int 2:121, 2011

10. Brown JM: The reconstructive neurosurgery of spinal cord injury, in Dimitrijevic MR, Kakulas BA, McKay WB, et al (eds): Restorative Neurology of Spinal Cord Injury. New York: Oxford University Press, 2012, pp 134-168

11. Brown JM, Vivio N, Sheean GL: The clinical practice of reconstructive neurosurgery. Clin Neurol Neurosurg 114:506-514, 2012

12. Coulet B, Allieu Y, Chammas M: Injured metamere and functional surgery of the tetraplegic upper limb. Hand Clin 18:399-412, vi, 2002

13. Hentz VR: Surgical strategy: matching the patient with the procedure. Hand Clin 18:503-518, 2002

14. Kozin SH: Tetraplegia. J Hand Surg Am 2:141-152, 2002

15. Leclercq C: Surgical rehabilitation for the weaker patients (groups 1 and 2 of the International Classification). Hand Clin 18:461-479, 2002 
16. Midha R: Nerve transfers for severe brachial plexus injuries: a review. Neurosurg Focus 16(5):E5, 2004

17. National Spinal Cord Injury Statistical Center: Facts and Figures at a Glance. (https://www.nscisc.uab.edu/ PublicDocuments/fact_figures_docs/Facts\%202013.pdf) [Accessed August 25, 2014]

18. Revol M, Cormerais A, Laffont I, Pedelucq JP, Dizien O, Servant JM: Tendon transfers as applied to tetraplegia. Hand Clin 18:423-439, 2002

19. Senjaya F, Midha R: Nerve transfer strategies for spinal cord injury. World Neurosurg 80:e319-e326, 2013

20. Squitieri L, Chung KC: Current utilization of reconstructive upper limb surgery in tetraplegia. Hand Clin 24:169-173, v, 2008

21. Zancolli EA: Midcervical tetraplegia with strong wrist extension: a two-stage synergistic reconstruction of the hand. Hand Clin 18:481-495, vii, 2002

\section{Response}

\section{Jayme Augusto Bertelli, MD, PhD, and Marcos Flávio Ghizoni, MD, MSc}

Department of Neurosurgery, University of the South of Santa Catarina (UNISUL), Tubarão, Brazil

Patients who suffer complete paralysis from a cervical SCI typically are referred to rehabilitation centers after both their overall clinical status and their spine are stabilized. There is always a hope for late spontaneous recovery, so surgical reconstruction of the upper limb generally is delayed. One crucial question is: how long should we wait for spontaneous recovery to plateau? For upperlimb reconstruction, the general rule is to wait at least 12 months. ${ }^{8}$ However, if we consider nerve transfers rather than tendon transfers, delayed reconstruction may lead to poorer results. Empirical evidence in the literature fails to support this 12-month waiting period. For instance, only $10 \%$ of patients with complete tetraplegia at the time of injury, without sacral sparing, exhibit any motor recovery 1 year later. ${ }^{4}$ Most spontaneous recovery of upperlimb function occurs within the first 6 months of an SCI. 7 There is virtually no chance that a muscle fully paralyzed 6 months after trauma will spontaneously regain active useful motor power over the next 6 months. ${ }^{7}$ Hence, we believe that 6 months is a sufficient waiting period.

Paralysis following SCI results from absent supraspinal control, in which case the muscles remain innervated, or from the death of motor neurons at the spinal cord level. This last condition reproduces a polio-like syndrome with Wallerian degeneration, mimicking a peripheral nerve injury, which warrants immediate repair to attain better results. In our study, we identified 22 peripheral palsies in 27 investigated nerves. Electromyograms can help to confirm muscle denervation; however, electromyographic signs of recovery do not necessarily reflect clinical improvement. ${ }^{5}$

Another question of interest is: are nerve transfers valid in chronic cases in which the muscles are paralyzed but not denervated (i.e., central palsy)? Brown ${ }^{1}$ reported on a patient operated on using nerve transfers 13 years after the injury. He demonstrated the feasibility of the technique, but did not report the results of upper-limb reconstruction. Mackinnon et al. ${ }^{3}$ reported on a patient who underwent an operation 23 months after injury for finger flexion reconstruction, transferring the nerve to the brachialis to the anterior interosseous nerve; in this case, the patient experienced modest recovery. This result motivated an investigator to write a letter to the editor of the Journal of Neurosurgery questioning the value of that procedure. ${ }^{2}$ We have operated on 4 patients with spinal cord lesions that were 3 to 6 years old from the time of their occurrence and have now followed them for 1 year postoperatively. While we did observe some recovery, it was only of a limited extent. Based on the concept that muscles remain innervated below the level of an SCI, in 2 patients with tetraplegia and chronic denervated palsies of the extensor digitorum communis muscle, we transferred a free gracilis muscle and innervated it using the nerve to the supinator to reconstruct thumb and finger extension. One year after surgery, some recovery was obtained, but it was not yet enough to identify any functional improvement. This clearly contrasts with the results we have obtained with the same surgery in the reconstruction of lower-type injuries of the brachial plexus. ${ }^{6}$ We do not yet have clear evidence that late nerve transfers are useful in longstanding SCIs. However, it is possible that these muscles, which are innervated but are functionally paralyzed for several years after reinnervation by a nerve transfer, take longer for recovery. Longer follow-up evaluation is needed before definitive conclusions can be made. As with any nerve repair, age matters substantially, and in our opinion, the patient's age should be taken into consideration before proceeding to late nerve versus tendon transfers.

Nerve transfers for elbow/thumb/finger extension reconstruction are reliable. Hence, nerve transfers should not be pitted against tendon transfers; rather they should be considered complementary. We insist on early nerve transfer surgery and believe that efforts should be made to recruit patients with tetraplegia earlier. Moreover, these concepts should be extended to rehabilitation centers.

\section{References}

1. Brown JM: Nerve transfers in tetraplegia I: background and technique. Surg Neurol Int 2:121, 2011

2. Fridén J, Gohritz A: Muscle and nerve transfer in tetraplegia. J Neurosurg 118:706-707, 2013 (Letter)

3. Mackinnon SE, Yee A, Ray WZ: Nerve transfers for the restoration of hand function after spinal cord injury. Case report. J Neurosurg 117:176-185, 2012

4. Marino RJ, Ditunno JF, Donovan WH, Maynard F: Neurologic recovery after traumatic spinal cord injury: data from the model spinal cord injury systems. Arch Phys Med Rehabil 80:1391-1396, 1999

5. Pitt M, Vredeveld JW: The role of electromyography in the management of the brachial plexus palsy of the newborn. Clin Neurophysiol 116:1756-1761, 2005

6. Soldado F, Bertelli J: Free gracilis transfer reinnervated by the nerve to the supinator for the reconstruction of finger and thumb extension in longstanding C7-T1 brachial plexus root avulsion. J Hand Surg Am 38:941-946, 2013

7. Waters RL, Adkins RH, Yakura JS, Sie I: Motor and sensory recovery following complete tetraplegia. Arch Phys Med Rehabil 74:242-247, 1993

8. Waters RL, Sie IH, Gellman H, Tognella M: Functional hand surgery following tetraplegia. Arch Phys Med Rehabil 77:86-94, 1996 